\title{
Aspectos biológicos e morfológicos de Leucanella viridescens (Lepidoptera: Saturniidae: Hemileucinae)
}

\author{
Alexandre Specht ${ }^{1,2}$; Elio Corseuil ${ }^{3}$; Augusto J. Benedetti ${ }^{\text {; }}$ Graziela Poletto ${ }^{1}$ \& Aline C. Formentini ${ }^{2}$ \\ ${ }^{1}$ Laboratório de Biologia, Centro de Ciências Exatas, da Natureza e de Tecnologia, Campus Universitário da Região dos \\ Vinhedos, Universidade de Caxias do Sul, Caixa Postal 32, 95700-000 Bento Gonçalves, Rio Grande do Sul, Brasil. \\ E-mail: aspecht@ucs.br \\ ${ }^{2}$ Instituto de Biotecnologia, Universidade de Caxias do Sul. Caixa Postal 1352, 95070-560 Caxias do Sul, Rio Grande do Sul, \\ Brasil. \\ ${ }^{3}$ Livre Docente em Entomologia. Professor Titular aposentado. E-mail: corseuil@via-rs.net
}

\begin{abstract}
Biological and morphological aspects of Leucanella viridescens (Lepidoptera: Saturniidae: Hemileucinae). The goal of this work was to describe aspects of the biology and morphology of Leucanella viridescens (Walker, 1855), a polyphytophagous species that is a pest to several crops of economical interest and whose larvae may cause erucism. Biological parameters were obtained under controlled conditions: temperature of $25 \pm 1{ }^{\circ} \mathrm{C}$, relative humidity of $70 \pm$ $10 \%$, and a $14 \mathrm{~h}$ photophase. Morphological and behavioral aspects were analyzed in each developmental phase. The larval stage had six instars, with an average growth rate of 1.396. A high degree of polyphytophagy was observed, including over 49 host plant species belonging to 25 families, especially Leguminosae, Solanaceae and Salicaceae. The considerable chromatic variability of the adults obtained from the same egg batch corroborates the need for a new taxonomic assessment of L. viridescens viridescens Walker, 1855 and L. viridescens viridior Lemaire, 1973.
\end{abstract}

KEY WORDS. Caterpillars; chromatic variation, erucism; life cycle; morphology.

Leucanella foi descrito por Lemaire (1969) e abriga 26 espécies anteriormente incluídas em Automeris Hübner, [1819] em função da notável homogeneidade dos hábitos, da morfologia geral dos adultos, especialmente da genitália e das formas imaturas (Lemaire 2002).

Entre os representantes mais freqüentemente encontrados no sul do Brasil e países limítrofes, figura Leucanella viridescens (Walker, 1855), cujos conhecimentos restringem-se a registros de ocorrência e de plantas hospedeiras (ex. Mabilde 1896, RonNA 1933a, b, Hambleton 1935, Costa Lima 1936, Biezanko \& Seta 1939, Biezanko et al. 1957, BAUCKe 1960, Silva et al. 1968, BiezANKo et al. 1974, Bertels 1975, Pastrana 2004, Specht et al. 2005), referência à ação urticante das cerdas de suas larvas (MORAEs 2003), descrição sumária de alguns aspectos biológicos, morfológicos (MABILDE 1896, Bourquin 1944) e ecológicos (Formentini et al. 2003). Lemaire (2002) detalha a morfologia dos adultos e a taxonomia.

Lemaire (1973) descreveu L. viridescens viridior para as populações localizadas mais ao oeste do que L. viridescens viridescens, baseando-se na ausência da coloração avermelhada característica da subespécie típica, principalmente da fêmea. Lemaire (2002) indica que essa subespécie ocorre da Bolívia até a Argentina (Buenos Aires) e Uruguai, enquanto que a subespécie típica é simpátrica podendo ser encontrada do Estado de Goiás, até Missiones, Argentina, incluindo Rio Grande do Sul. Segundo Lemaire (2002), a variação da coloração dos adultos gerou ainda sinonímias errôneas com Leucanella memusae (Walker, 1855) (e.g. Draudt 1929, KÖHLer 1935, Bouvier 1936) e a descrição de "formas" por SCHÜssLer (1934).

Através de uma criação em laboratório, o presente estudo pretende contribuir com conhecimentos biológicos e morfológicos, considerando principalmente a variação cromática dos adultos.

\section{MATERIAL E MÉTODOS}

A criação foi realizada em sala climatizada sob temperatura de $25 \pm 1^{\circ} \mathrm{C}$, umidade relativa de $70 \pm 10 \%$ e fotofase de 14 horas, com observações diárias. As formas imaturas de $L$. viridescens foram obtidas a partir de duas posturas provenientes de uma fêmea coletada em Bento Gonçalves, Rio Grande do Sul, em 20 de maio de 2006.

Os aspectos morfológicos dos imaturos foram avaliados efetuando-se mensurações através de paquímetro digital, balança analítica e microscópio estereoscópico provido de ocular micrométrica.

As posturas foram individualizadas em placas de Petri contendo papel filtro umedecido com água destilada, onde permaneceram até a eclosão das larvas. Além de observações morfológicas, foram efetuadas mensurações do comprimento, eixo entre a área micropilar e a porção fixa ao substrato e da maior e menor largura em eixos perpendiculares ao primeiro. 
Desde a eclosão até o período de pré-pupa, as larvas provenientes de cada postura foram mantidas em gaiolas teladas com dimensões de $80 \mathrm{~cm}$ de altura, $50 \mathrm{~cm}$ de largura e $42 \mathrm{~cm}$ de profundidade. Foram alimentadas com folhas de corticeirado-banhado, Erythrina crista-galli Linnaeus (Fabaceae), disponibilizada em ramos, renovados diariamente, cujas extremidades foram introduzidas em frascos de $50 \mathrm{ml}$ com água destilada, para manter a turgescência. Durante as atividades de manutenção, foram recolhidas as cápsulas cefálicas para posterior medida da distância entre as cerdas frontais (Podoler \& KLeIN 1978). Os ínstares foram diferenciados conforme referido por PARRA \& HADDAD (1989), utilizando a distribuição de freqüência das medidas entre as cerdas frontais, em intervalos de décimo de milímetro, para posterior agrupamento; ao final, elaborouse uma curva de distribuição de freqüência, ajustada ao modelo de Dyar (1890).

No período de pré-pupa, quando pararam de se alimentar e se dispersaram procurando um local para a construção do casulo, entre as folhas e demais estruturas vegetais, as larvas foram individualizadas em recipientes de vidro com capacidade de $500 \mathrm{ml}$, com papel filtro umedecido com água destilada no fundo e tapados com "voile".

No segundo dia após a formação, quando a cutícula já estava endurecida, os casulos foram abertos e as pupas foram removidas para a verificação do sexo, conforme ButT \& CANTU (1962); foram avaliados o peso, a maior largura e o comprimento.

Após a emergência, os adultos foram sacrificados em congelador a $-17^{\circ} \mathrm{C}$ e, posteriormente, distendidos em extensores. Avaliou-se a envergadura, expressa pela soma do comprimento das asas anteriores e a largura torácica entre os seus pontos de inserção.

Como material testemunho, conservou-se em álcool etílico, 70\% das posturas, 10 larvas de último ínstar e 10 pupas (cinco de cada sexo); também foram conservados a seco, 13 machos e 14 fêmeas. Todo o material encontra-se depositado na coleção do Laboratório de Biologia (CUCS), Campus Universitário da Região dos Vinhedos, Universidade de Caxias do Sul (CARVI-UCS).

Os dados morfométricos e de massa foram analisados, calculando-se médias e erros-padrões; as comparações morfométricas e de massa entre sexos, das pupas e dos adultos, foram realizadas pelo teste " $\mathrm{t}$ ", ao nível de significância de 95\%.

Além da criação laboratorial, realizou-se um inventário das plantas hospedeiras citadas em bibliografia, cuja nomenclatura foi atualizada, principalmente, conforme BACKES \& NARDINO (2001), relacionando-se os nomes vulgares, científicos, família e referências (Tab. I).

Tabela I. Plantas hospedeiras de Leucanella viridescens.

\begin{tabular}{|c|c|c|c|}
\hline Nome popular & Nome científico & Família & Referências \\
\hline Abacateiro & Persea americana Mill. & Lauraceae & 13,15 \\
\hline Acácia-comum & Acacia longifolia Willd. & Leguminosae & 15 \\
\hline Acácia-negra & Acacia mearnsii De Willd. & Leguminosae & 15 \\
\hline Álamo & Populus nigra Linn. & Salicaceae & $11,13,15$ \\
\hline Álamo-branco & Populus alba pyramidalis (Bunge) W. Wett. & Salicaceae & 14,15 \\
\hline Álamo-da-carolina & Populus deltoides Marsh. & Salicaceae & $11,13,15$ \\
\hline Álamo-do-canadá & Populus canadensis Moench & Salicaceae & $11,13,15$ \\
\hline Álamo-preto & Populus italica Derroi & Salicaceae & $11,13,15$ \\
\hline Arecas & - & Arecaceae & 13,15 \\
\hline Aroeira-vermelha & Schinus terebinthifolius Raddi & Anacardiaceae & 13,15 \\
\hline Batata-doce & Ipomea batatas (Linn.) Lam. & Convolvulaceae & 14,15 \\
\hline Batatinha & Solanum tuberosum Linn. & Solanaceae & $9,10,11,13,15$ \\
\hline Berinjela & Solanum melongena Linn. & Solanaceae & $10,11,13,14,15$ \\
\hline Candiúba & Trema micrantha (Linn.) Blume & Ulmaceae & $5,10,15$ \\
\hline Cinamomo & Melia azedarach Linn. & Meliaceae & $8,11,13,14,15$ \\
\hline Coerana & Cestrum parqui L'Herit. & Solanaceae & $11,13,14,15$ \\
\hline Coronilha & Scutia buxifolia Reiss. & Rhamnaceae & 13 \\
\hline Corticeira-da-serra & Erythrina falcata Benth & Leguminosae & 15 \\
\hline Corticeira-de-jardim & Erythrina speciosa Andrews & Leguminosae & 15 \\
\hline Corticeira-do-banhado & Erythrina crista-galli Linn. & Leguminosae & $1,2,6,7,8,9,10,11,13,14,15$ \\
\hline
\end{tabular}


Tabela I.Continuação.

\begin{tabular}{|c|c|c|c|}
\hline Nome popular & Nome científico & Família & Referências \\
\hline Erva-mate & Ilex paraguariensis St. Hil. & Aquifoliaceae & $9,10,13,14,15$ \\
\hline Fumo-bravo & Solanum auriculatum (Aiton) O. Kuntze & Solanaceae & $11,13,15$ \\
\hline Goiabeira & Psidium guajava Linn. & Myrtaceae & 13,15 \\
\hline Guaxuma & Sida rhombifolia Linn. & Malvaceae & 13,15 \\
\hline Hera & Hedera helix Linn. & Araliaceae & 13,15 \\
\hline Jacarandá & Jacaranda mimosaefolia D. Don. & Bignoniaceae & $9,10,13,15$ \\
\hline Japecanga & Smilax campestris Griseb. & Smilacaceae & $1,3,9,10,15$ \\
\hline Jurubeba & Solanum bonariense Linn. & Solanaceae & $11,13,14,15$ \\
\hline Jurubeba-da-boa & Solanum variabile Mart. & Solanaceae & $9,14,15$ \\
\hline Jurubeba-verdadeira & Solanum paniculatum Linn. & Solanaceae & 14 \\
\hline Kiwi & Actinidia chinensis Planch & Actinidiaceae & 13,15 \\
\hline Ligustro & Ligustrum lucidum Ait. & Oleaceae & 13,15 \\
\hline Língua-de-vaca & Rumex crispus Linn. & Polygonaceae & 13,15 \\
\hline Madressilva & Lonicera japonica Thumb. & Caprifoliaceae & $1,8,9,10,11,13,15$ \\
\hline Madressilva-caprifolia & Lonicera caprifolia Linn. & Caprifoliaceae & 10,14 \\
\hline Madressilva-trombeta & Lonicera sempervirens Linn. & Caprifoliacae & 14 \\
\hline Maria-mole & Senecio brasiliensis (Spreng.) ex Backer & Asteraceae & 13,15 \\
\hline Mata-pasto & Vernonia polyanthes Less. & Asteraceae & 13,15 \\
\hline Milho & Zea mayz Linn. & Poaceae & 13,15 \\
\hline Mimo-de-vênus & Hibiscus rosa-sinensis Linn. & Malvaceae & 13,15 \\
\hline Oliveira & Olea europea Linn. & Oleaceae & $4,8,9,10,11,13,14,15$ \\
\hline Pelargonio & Pelargonium sp. & Geraniaceae & 15 \\
\hline Pereira & Pyrus communis Linn. & Rosaceae & $6,9,10,13,14,15$ \\
\hline Pessegueiro & Prunus persica (L.) Batsch & Rosaceae & 13,15 \\
\hline Salseiro & Salix humboldtiana Willd. var. martiana Anders & Salicaceae & 14 \\
\hline Samambaia-do-campo & Pteridium aquilinum (L.) Kuhn & Polypodiaceae & 13,15 \\
\hline Sarandi & Cephalanthus sarandi Cham. \& Schlecht. & Rubiaceae & $1,9,10,14,15$ \\
\hline Soja & Glycine max (Linn.) Merr. & Leguminosae & 12,14 \\
\hline Unha-de-Vaca & Bauhinia forficata Link. & Leguminosae & 13,15 \\
\hline
\end{tabular}

Com vistas a obter dados sobre a época de ocorrência dos adultos, foram examinados exemplares coletados no Rio Grande do Sul e depositados em coleções sediadas no mesmo estado: Centro de Pesquisa Agropecuária de Clima Temperado (CAMB), Museu de Ciências da Universidade Católica de Pelotas (MUCP) e Museu Entomológico Ceslau Biezanko (MECB), em Pelotas; Museu Anchieta de Porto Alegre (MAPA), Museu de Ciências e Tecnologia da PUCRS (MCTP), Museu de Ciências Naturais da Fundação Zoobotânica do Rio Grande do Sul (MCNZ), Museu Ramiro Gomes Costa da Fundação Estadual de
Pesquisa Agropecuária (MRGC), em Porto Alegre e coleção do próprio Laboratório (CUCS), em Bento Gonçalves.

\section{RESULTADOS E DISCUSSÃO}

\section{Ovo}

Os ovos (Figs 1-2), de formato ovóide levemente comprimido, ficam aderidos ao substrato pelo pólo mais estreito, oposto à micrópila, que é mais plano. Coloração, incluindo a micrópila, verde-clara, diferindo do observado por BouRquin (1944), que os refere como brancos. A distância entre a micrópila e a extremida- 

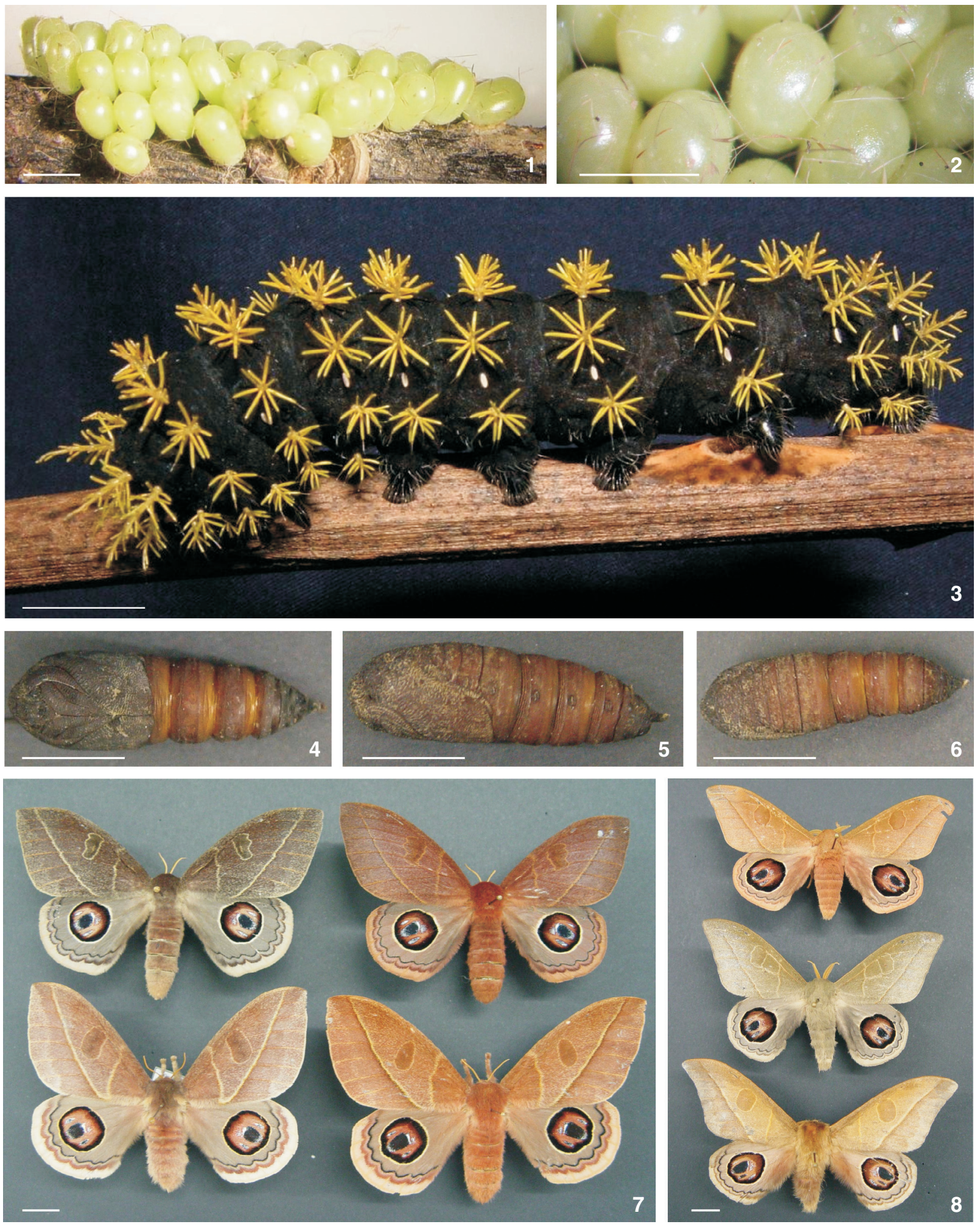

Figuras 1-8. Leucanella viridescens: (1) postura; (2) detalhe de ovos; (3) larva de último instar; (4) pupa em vista ventral; (5) pupa em vista lateral; (6) pupa em vista dorsal; (7) fêmeas; (8) machos. Barra $=1 \mathrm{~cm}$.

ZOOLOGIA 26 (1): 25-31, March, 2009 
de oposta é 6,647 $\pm 0,089 \mathrm{~mm}$, a maior e menor largura foram $4,853 \pm 0,118 \mathrm{~mm}$ e $3,881 \pm 0,112 \mathrm{~mm}$, respectivamente $(\mathrm{n}=20$ ). Estes resultados mostram que, entre os representantes do gênero, há uma grande variedade de formas e de colorações dos ovos. Lemaire (2002) cita que, entre as poucas espécies com biologia conhecida, encontram-se ovos de formato oval, na maioria brancos com micrópila preta ou, como os de L. aspera (R. Felder \& Rogenhofer, 1874), verdes logo após a postura, tornando-se amarelos no dia seguinte. A coloração dos ovos, próximo à eclosão das larvas, vai escurecendo até tornar-se praticamente negra internamente, com o cório opaco-esbranquiçado.

\section{Larva}

Observaram-se seis ínstares larvais (Tab. II) com uma razão média de crescimento de 1,396 para as cápsulas cefálicas, entre os ínstares, sendo coerente com o previsto pela regra de DyAr (1890). O tamanho da cápsula cefálica variou de 0,798 $\mathrm{mm}$ até 4,312 $\mathrm{mm}$ do primeiro ao último instar, semelhante ao descrito para representantes de Automeris Hübner, [1819] (SРеснт et al. 2005, 2006a, 2007). Em diversas ocasiões em que houve contato acidental com as larvas, observou-se que são urticantes desde o primeiro ínstar, concordando com as observações de Bourquin (1944) e de Moraes (2003). Entretanto, os efeitos urticantes nunca duraram mais do que três horas.

Da eclosão até o terceiro instar, observou-se gregarismo acentuado em toda criação mantida em laboratório, com lar-

Tabela II. Médias da distância ( $\mathrm{mm})$, entre cerdas frontais com respectivos erros padrões e razão de crescimento de larvas de Leucanella viridescens alimentadas com folhas de corticeira-dobanhado (Erythrina crista-galli), a $25 \pm 1^{\circ} \mathrm{C}$, UR $70 \pm 10 \%$ e 14 horas de fotofase.

\begin{tabular}{cccc}
\hline Instar & $\mathrm{n}$ & Média \pm erro-padrão & Razão de crescimento \\
\hline I & 83 & $0,093 \pm 0,002$ & - \\
II & 68 & $0,138 \pm 0,003$ & 1,484 \\
III & 63 & $0,196 \pm 0,005$ & 1,420 \\
IV & 58 & $0,273 \pm 0,008$ & 1,393 \\
V & 49 & $0,375 \pm 0,021$ & 1,374 \\
VI & 42 & $0,490 \pm 0,034$ & 1,307 \\
\hline
\end{tabular}

vas ocupando uma mesma folha, alimentando-se concomitantemente, o que é comum entre os representantes de Hemileucinae (Lemaire 2002). Do quarto ínstar em diante, as larvas começaram a formar grupos menores e no último, diversas dispersaram-se individualmente.

Desde o primeiro ínstar até o final do desenvolvimento, as larvas apresentam a coloração de fundo e a cabeça negras. Entretanto, no primeiro instar, as cerdas são amarelo pálido, tornando-se mais intenso nos ínstares seguintes. Algumas larvas, especialmente logo após a ecdise, apresentam as cerdas amarelo-limão intenso. Nos dois primeiros ínstares, as larvas apresentam os escolos de tamanhos diferenciados, como descrito para a maioria dos hemileucíneos (Lemaire 2002); entretanto, a partir do terceiro instar, tornam-se menos diferenciados e relativamente curtos (Fig. 3), notando-se, especialmente, que os escolos dorsais do pró e mesotórax e do nono urômero são ligeiramente mais desenvolvidos.

A reunião das plantas hospedeiras (Tab. I) permitiu relacionar 49 táxons, pertencentes a 25 famílias, destacando-se Leguminosae e Solanaceae, com sete espécies, e Salicaceae com seis. L. viridescens, como diversos outros hemileucíneos dos gêneros Automeris (Stone 1991, Lemaire 2002, Specht et al. 2005, 2006a, 2007), Hylesia Hübner, [1820] (SРеCHT et al. 2005, 2006b) e Hyperchiria Hübner, [1819] (SРЕсHT et al. 2005), é polifitófago. Além disso, como em Automeris illustris (Walker, 1855), FormenTINI et al. (2003) observaram que suas larvas dificilmente mudam de planta hospedeira durante o desenvolvimento. Assim, quando as larvas eram coletadas em campo e havia a indicação da planta hospedeira, FormenTINI et al. (2003) puderam continuar a alimentação até o final da fase, mas sem trocar de planta, a não ser que fosse alguma espécie de corticeira. Isto indica que, apesar da polifitofagia, estes lepidópteros experimentam, quando iniciam a alimentação desde o início em uma determinada planta, uma indução à preferência específica. Desta forma, segundo JANZEN (2003), esta espécie pode ser enquadrada no grupo que se alimenta de cinco a 10 espécies de plantas aparentadas até completamente não relacionadas em um ecossistema natural, mas cada larva individualmente alimenta-se apenas da planta hospedeira utilizada pelo adulto para ovipositar. FormenTINI et al. (2003) salientam que quando foram trazidas larvas de $L$. viridescens, sem indicação de planta hospedeira, sempre que se ofereceu alguma espécie do gênero Erythrina, houve aceitação imediata, corroborando sua preferência por E. crista-galli, já descrita em bibliografia (Mabilde 1896, BieZanko \& Seta 1939, Bourquin 1944).

Segundo Formentini et al. (2003), a grande ocorrência de L. viridescens, bem como dos outros hemileucíneos polifitófagos, nos ambientes ocupados pelos humanos está relacionada com sua grande plasticidade ecológica, aqui discutida apenas sob o ponto de vista da capacidade de adaptação às plantas hospedeiras de diversos grupos vegetais, especialmente as cultivadas.

No período de pré-pupa, as larvas reduziram de tamanho, pararam a atividade de alimentação e procuraram um lugar para a confecção do casulo. Confeccionaram envoltórios de parede única, resistentes e impermeáveis, não bem caracterizados como casulos, utilizando fios grosseiros de seda de cor âmbar, unindo folhas conforme descrito por BoURQUIN (1944), assemelhando-se muito aos descritos por Lemaire (2002) para espécies de Automeris. Todas as 61 pré-pupas transformaram-se em pupas sem deformação.

\section{Pupa}

As pupas (Figs 4-6) femininas $(\mathrm{n}=27$ ) foram significativamente maiores que as masculinas $(n=34)$, com pesos de $2,518 \pm 0,131$ g e 1,691 $\pm 0,084$ g respectivamente; comprimentos de 32,641 $\pm 0,290 \mathrm{~mm}$ e $28,501 \pm 0,204 \mathrm{~mm}$ e larguras de $11,816 \pm 0,175 \mathrm{~mm}$ e $10,572 \pm 0,125 \mathrm{~mm}$ respectivamente. 
Tais diferenças relacionam-se ao grande dimorfismo sexual, característico do grupo, também relatado para as outras espécies de Leucanella (Lemaire 2002).

\section{Adulto}

Das 61 pupas emergiram 26 fêmeas e 33 machos, apresentando grande variabilidade cromática (Figs 7 e 8), relacionada ao dimorfismo sexual característico dos hemileucíneos e entre indivíduos do mesmo sexo, mesmo quando provenientes de uma mesma postura. Desta forma, a distinção das subespécies $L$. viridescens viridescens e $L$. viridescens viridior, baseada na coloração das fêmeas (Lemaire 2002), deve ser revista.

As fêmeas foram significativamente maiores, com envergadura média de $91,374 \pm 1,708 \mathrm{~mm}(\mathrm{n}=12)(99,886$ a 89,654 $\mathrm{mm})$, enquanto a dos machos foi de 78,776 $\pm 1,504 \mathrm{~mm}(\mathrm{n}=$ 14) $(87,530$ a $66,872 \mathrm{~mm})$. As variações da envergadura assemelham-se e abrangem os valores relacionados para as duas subespécies (Lemaire 2002).

A reunião dos dados dos exemplares depositados em coleção (Fig. 9) demonstrou que os adultos podem ser coletados praticamente durante todo o ano, exceto nos meses de maio e junho. A ausência de adultos nos meses mais frios indica que, conforme sugerido por BourQuin (1944), L. viridescens sobrevive ao inverno na fase de pupa. Além disso, a reunião dos dados indicou a existência de duas épocas de maior ocorrência dos adultos: a primeira em fevereiro e uma segunda entre agosto e outubro, semelhante ao observado para outras espécies de Hemileucinae ocorrentes no Rio Grande do Sul (Specht et al. 2006a, 2007).

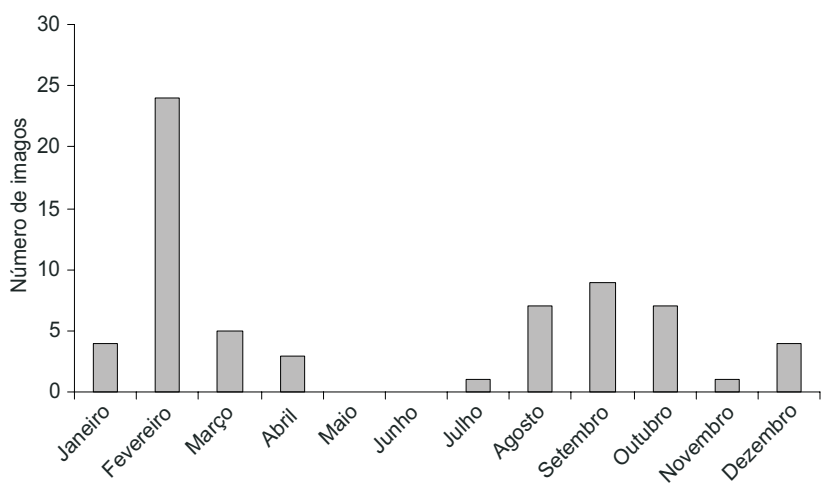

Figura 9. Número de imagos de Leucanella viridescens capturados no Rio Grande do Sul, segundo meses de coleta.

Registra-se a ocorrência do parasitóide larval Enicospilus sp. (Hymenoptera: Ichneumonidae), em função de três exemplares (MUCP), obtidos de criação conduzida pelas irmãs Figueiredo, em 10.II.1945.

\section{AGRADECIMENTOS}

Aos curadores Eduardo J.E. Silva (MECB), Élvia E.S. Vianna (MUCP), Fernando R. Meyer (MAPA), Luciano Moura (MCNZ),
Mirtes Melo (CAMB) e Vera R.S. Wollf (MRGC) pelo acesso e auxílio prestado durante o exame do material. À UCS, ao CNPq e à FAPERGS, pela concessão das Bolsas de Iniciação Científica aos três últimos autores. À FAPERGS pelo Auxílio Financeiro (Processo 02/1739.6). À Liane T. Dorneles pela foto da larva de último instar.

\section{LITERATURA CITADA}

Backes, A. \& M. NARdino. 2001. Nomes populares e científicos de plantas do Rio Grande do Sul. São Leopoldo, Unisinos, Coleções Fisionomia Gaúcha, $2^{a}$ ed., 202p.

BAUCKE, O. 1960. Notas entomológicas III. O gênero Automeris Hübner, 1819 (Lepidoptera, Hemileucidae) no Rio Grande do Sul. Iheringia, Série Zoologia, 12: 11-19.

Bertels, A.M. 1975. Insetos-pragas da soja e seu combate. Pelotas, Embrapa, Boletim Técnico, 100, 33p.

Biezanko, C.M. \& F.D. Seta. 1939. Catálogo dos insetos encontrados em Rio Grande e seus arredores. Pelotas, Universal, $15 \mathrm{p}$.

Biezanko, C.M.; Ruffinelli, A. \& C.S. Carbonell. 1957. Lepidoptera Del Uruguay - Lista anotada de espécies. Revista de la Faculdad de Agronomía 46: 1-152.

Biezanko, C.M.; A. Ruffinelli \& D. Link. 1974. Plantas y otras sustancias alimenticias de las orugas de los lepidópteros uruguayos. Revista do Centro de Ciências Rurais 4 (2): 107-148.

Bourquin, F. 1944. III - Observaciones sobre Automeris viridescens (Walker) 1855. (Lep. Fam. Hemileucinae), p. 9-11. In: F. BouRQuIN (Ed.). Mariposas Argentinas: Vida, desarrolo, costumbres y hechos curiosos de algunos lepidópteros argentinos. Buenos Aires, Ferrari Hermanos Bartolome Mitre, 213p.

Bouvier, E.L. 1936. Étude des Saturnioïdes normaux. Familie des Hémileucidés. Trosième et dernière partie. Annales de Sciences Naturelles et de Zoologie 10 (19): 267-529.

ButT, B.A.\& E. CANTU. 1962. Sex determination of lepidopterous pupae. Washington, USDA, 7p.

Costa Lima, A.M. 1936. Terceiro catálogo dos insetos que vivem nas plantas do Brasil. Rio de Janeiro, Ministério da Agricultura, Escola Nacional de Agronomia, 460p.

Draudt, M. 1929-1930. 12. Familie Saturniidae, p. 713-827. In: A. Seitz (Ed.). Die Gross-Schmetterlinge der Erde. 5. Die Amerikanischen Spinner und Schwärmer. Stuttgart, Alfred Kernen, 508p.

Dyar, H.G. 1890. The number of molts of lepidopterous larvae. Psyche 5: 420-422.

Formentini, A.C.; A. Specht; L.T. Dorneles; E. Corseuil \& H.B. Abella. 2003. Aspectos ecológicos de Leucanella viridescens (Walker, 1855) (Lepidoptera: Saturniidae: Hemileucinae) no Rio Grande do Sul, p. 42-43. In: V. Claudino-Sales; J.M. Tonini $\&$ E.W.C. Dantas (Eds). 1. Ecossistemas brasileiros. Fortaleza, Editora da Universidade Federal do Ceará, 613p.

Hambleton, E.J. 1935. Alguns dados sôbre lepidópteros brasileiros do Estado de Minas Geraes. Revista de Entomologia 5 (1): 1-7. 
JANZEN, D.H. 2003 How polyphagous are Costa Rican dry forest saturniid moth caterpillars?, p. 369-379. In: Y. BASSET; R. Kitching; S. Miller \& V. Novotny (Eds). Arthropods of Tropical Forests: spatio-temporal dynamics and resource use in the canopy. Cambrigde, Cambridge University Press, 490p.

KöHLER, P. 1935. Notas sobre Automerinae [sic] (Lep. Saturn.) Argentinos. Revista de la Sociedad Entomológica Argentina 7: 79-91.

Lemaire, C. 1969. Description de quatre genres nouveaux et d'une espècie inèdite d'"Hemileucinae" (Lep. Attacidae). Lambillionea 68: 39-52.

Lemaire, C. 1973. Révision du genre Automeris Hübner et des genres voisins. Biogéographie, éthologie, morphologie, taxonomie (Lep. Attacidae). Memoirs du Museum National d'Histoire Naturelle (France), Nouvelle Serie, Serie A. Zoologie 79: 233-422.

Lemaire, C. 2002. The Saturniidae of America Hemileucinae. Keltern, Goecke \& Evers, 1388.

Mabilde, A.P. 1896. Guia práctico para os principiantes collecionadores de insectos, contendo a descrição fiel de perto de 1000 borboletas com 180 figuras lytographadas em tamanho, formas e desenhos conforme o natural. Estudo sobre a vida de insectos do Rio Grande do Sul e sobre a caça, classificação e conservação de uma Coleção mais ou menos regular. Porto Alegre, Gunlach \& Schuldt, 238p.

Moraes, R.H.P. 2003. 21. Lepidópteros de importância médica, p. 211-219. In: J.L.C. Cardoso; F.O.S. França; F.H. Wen; C.M.S. Málaque \& V. Haddad Jr (Eds). Animais peçonhentos no Brasil - Biologia, clínica e terapêutica dos acidentes. São Paulo, Sarvier, 468p.

PARRA, J.R.P.\& M.L. HADDAD. 1989. Determinação do número de ínstares de insetos. Piracicaba, FEALQ, 49p.

Pastrana, J.A. 2004. Los lepidópteros argentinos: sus plantas hospedadoras y otros sustratos alimenticios. Buenos Aires, Sociedad Entomológica Argentina, 350p.Podoler, H. \& M. KLEIN. 1978. Distance between frontal setae: a new tool for determining caterpillar instars. Journal of Natural History 12 (3): 341-347.

Ronna, E. 1933a. Catálogo dos insetos até hoje encontrados nas plantas do Rio Grande do Sul. Egatéa 18 (4): 197-202.

RonnA, E. 1933b. Catálogo dos insetos até hoje encontrados nas plantas do Rio Grande do Sul. Egatéa 18 (6): 329-334.

SCHÜssLER, H. 1934. Saturniidae: 2 Subfam. Saturniinae II et 3. Subfam. Ludiinae I, p. 325-484. In: E. Strand (Ed.). Lepidopterorum Catalogus, Pars. 58. Berlin, W. Junk, 769p. Silva, A.G.A.; Gonçalves, C.R.; Galvão, D.M.; Gonçalves, A.J.L., Gomes, J.; Silva, M.N. \& L. Simoni. 1968. Quarto catálogo dos insetos que vivem nas plantas do Brasil: seus parasitos e predadores. Rio de Janeiro, Ministério da Agricultura, $622 \mathrm{p}$.

Specht, A.; Corseuil, E. \& A.C. Formentini. 2005. Lepidópteros de importância médica ocorrentes no Rio Grande do Sul. III. Saturniidae, Hemileucinae. Biociências 13 (2): 149-162.

Specht, A.; A.C. Formentini \& E. Corseuil. 2006a. Biologia de Automeris illustris (Walker) (Lepidoptera, Saturniidae, Hemileucinae). Revista Brasileira de Zoologia 23 (2): 537546.

Specht, A.; A.C. Formentini \& E. Corseuil. 2006b. Biologia de Hylesia nigricans (Berg) (Lepidoptera, Saturniidae, Hemileucinae). Revista Brasileira de Zoologia 23 (1): 248-255.

Specht, A.; Formentini, A.C. \& E. Corseuil. 2007. Biologia e aspectos morfológicos dos estágios imaturos de Automeris naranja Schaus (Lepidoptera, Saturniidae, Hemileucinae). Revista Brasileira de Zoologia, 24 (3): 537-545.

Stone, S.E. 1991. Foodplants of World Saturniidae. Memoirs of the Lepidopteroist's Society 4: 1-186.

Submitted: 23.I.2008; Accepted: 02.II.2009.

Editorial responsibility: Gabriel Mejdalani 